\title{
NON-EXISTENCE, EXISTENCE AND UNIQUENESS OF LIMIT CYCLES FOR QUADRATIC POLYNOMIAL DIFFERENTIAL SYSTEMS
}

\author{
JAUME LLIBRE $^{1}$ AND XIANG ZHANG ${ }^{2}$
}

\begin{abstract}
We provide sufficient conditions for the non-existence, existence and uniqueness of limit cycles surrounding a focus of a quadratic polynomial differential system in the plane.
\end{abstract}

\section{Introduction AND STATEMENT OF the MAin RESUlts}

One of the main problems in the qualitative theory of real planar differential systems is to control the existence, non-existence and uniqueness of limit cycles for a given class of polynomial differential systems.

Limit cycles of planar differential systems were defined by Poincaré [15], and started to be studied intensively at the end of the 1920s by van der Pol [16], Liénard [11] and Andronov [1].

It is well known that if a quadratic polynomial differential system, or simply a quadratic system has one limit cycle this must surround a focus of the system (see for instance Proposition 8.13 of [7]), and according with Bautin [2] such a system can be written in the form

$$
\begin{aligned}
& \dot{x}=\lambda_{1} x-y-\lambda_{3} x^{2}+\left(2 \lambda_{2}+\lambda_{5}\right) x y+\lambda_{6} y^{2}, \\
& \dot{y}=x+\lambda_{1} y+\lambda_{2} x^{2}+\left(2 \lambda_{3}+\lambda_{4}\right) x y-\lambda_{2} y^{2} .
\end{aligned}
$$

In order to state our results we wrote the quadratic system (1) in polar coordinates $(r, \theta)$, defined by $x=r \cos \theta, y=r \sin \theta$, and we get

$$
\begin{aligned}
& \dot{r}=\lambda_{1} r+f r^{2}, \\
& \dot{\theta}=1+g r,
\end{aligned}
$$

where

$$
\begin{aligned}
f=f(\theta)= & -\lambda_{3} \cos ^{3} \theta+\left(3 \lambda_{2}+\lambda_{5}\right) \cos ^{2} \theta \sin \theta+ \\
& \left(2 \lambda_{3}+\lambda_{4}+\lambda_{6}\right) \cos \theta \sin ^{2} \theta-\lambda_{2} \sin ^{3} \theta, \\
g=g(\theta)= & \lambda_{2} \cos ^{3} \theta+\left(3 \lambda_{3}+\lambda_{4}\right) \cos ^{2} \theta \sin \theta- \\
& \left(3 \lambda_{2}+\lambda_{5}\right) \cos \theta \sin ^{2} \theta-\lambda_{6} \sin ^{3} \theta,
\end{aligned}
$$

2010 Mathematics Subject Classification. 34C07; 34C05.

Key words and phrases. Quadratic systems, quadratic polynomial differential system, limit cycles. 
are homogeneous polynomials of degree 3 in the variables $\cos \theta$ and $\sin \theta$. In the region

$$
C=\{(x, y)=(r \cos \theta, r \sin \theta): r \geq 0 \text { and } 1+g r>0\}
$$

the differential system (2) is equivalent to the differential equation

$$
\frac{d r}{d \theta}=\frac{\lambda_{1} r+f r^{2}}{1+g r}
$$

We note that $C$ is a simply connected region containing the origin of coordinates, having as boundary the points that in polar coordinates $(r, \theta)$ satisfy the equality $r=-1 / g$, i.e. the points of the curve $\dot{\theta}=0$.

It is known that the periodic orbits surrounding the origin of system (2) do not intersect the curve $\dot{\theta}=1+g r=0$ (see the Appendix of [4]). Therefore, these periodic orbits are contained in the region $C$, and consequently also they are periodic orbits of equation (4). Moreover these periodic orbits can be studied doing the change of variables

$$
\rho=\frac{r}{1+g(\theta) r}
$$

due to Cherkas [5]. In the new variable $\rho$ the differential equation (4) writes

$$
\frac{d \rho}{d \theta}=g\left(\lambda_{1} g-f\right) \rho^{3}+\left(f-2 \lambda_{1} g-g^{\prime}\right) \rho^{2}+\lambda_{1} \rho .
$$

Quadratic systems have been investigated intensively, and there are more than one thousand of articles published on themselves, and many of them on their limit cycles, see for instance $[17,20]$. But in this work we are interested in the results on the limit cycles of quadratic systems which have been obtained using the trigonometric polynomials $f$ and $g$ which appear in the differential equation (4). As far as we know those results are the following:

(I) If $g\left(\lambda_{1} g-f\right) \geq 0$ for all $\theta$, then the quadratic system (1) has at most one limit cycle surrounding the origin (see statement (b) of Theorem 1.1 of [3]).

(II) If $f-2 \lambda_{1} g-g^{\prime}=0$, then the quadratic system (1) has at most one limit cycle surrounding the origin (see statement (b) of Theorem $\mathrm{C}$ of [9]).

We must mention that results (I) and (II) where proved for polynomial differential systems more general than the quadratic ones, and that the versions presented here are their restriction to quadratic systems. As we shall see the result (I) continues be optimal for quadratic systems, in the sense that as we shall prove there are quadratic systems satisfying their assumptions and having either zero, or one limit cycles 
surrounding the origin of system (1). But the result (II) is not optimal for quadratic systems, because we shall prove that all quadratic systems under the assumptions of (II) have no limit cycles.

Now we state our results on the limit cycles of the quadratic systems using the trigonometric polynomials $f$ and $g$. We also include for completeness the known result (I).

Theorem 1. The quadratic system (1) has no limit cycles surrounding the origin if one of the following conditions hold:

(i) if $f=0$;

(ii) if $f-\lambda_{1} g=0$;

(iii) if $g=0$;

(iv) if $f-2 \lambda_{1} g-g^{\prime}=0$;

(v) if $\lambda_{1} g-9 f=0$;

(vi) if $9 \lambda_{1} g-f=0$;

(vii) if $\left(\lambda_{1} g-f\right)\left(\lambda_{1} g-9 f\right) \leq 0$ for all $\theta$.

The quadratic system (1) has at most one limit cycle surrounding the origin if one of the following conditions hold:

(viii) if $\left(\lambda_{1} g-f\right)\left(9 \lambda_{1} g-f\right) \leq 0$ for all $\theta$ and $\left(\lambda_{1} g-f\right)\left(9 \lambda_{1} g-f\right) \not \equiv 0$;

(ix) if $g\left(\lambda_{1} g-f\right) \geq 0$ for all $\theta$ and $g\left(\lambda_{1} g-f\right) \not \equiv 0$.

Theorem 1 is proved in section 3 .

We remark that condition (viii) cannot be obtained from (vii) by taking $9 \lambda_{1}$ instead of $\lambda_{1}$, because doing this to system (21), for the new system with $9 \lambda_{1}$ instead of $\lambda_{1}$ we have

$$
\left(\lambda_{1} g-f\right)\left(9 \lambda_{1} g-f\right)=\frac{11\left(9 \lambda_{1} \cos \theta-5 \sin \theta\right)^{2}}{81 \lambda_{1}^{2}} \geq 0,
$$

whereas

$$
\left(\lambda_{1} g-f\right)\left(\lambda_{1} g-9 f\right)=-\frac{\left(9 \lambda_{1} \cos \theta-5 \sin \theta\right)^{2}}{81 \lambda_{1}^{2}} \leq 0 .
$$

It follows easily from Theorem 1 the next result.

Corollary 2. If the inequalities of statements (viii) and (ix) are strict, then there exists an open set in the space of twelve coefficients of the quadratic systems where the corresponding quadratic systems have at most one limit cycle.

In the next result we show that there are quadratic systems satisfying all the statements of Theorem 1 with except statement (viii).

Proposition 3. We provide examples of quadratic systems satisfying the assumptions of all statements of Theorem 1, and we present examples of quadratic systems satisfying the assumption of statement (viii) 
with one limit cycle, and the assumption of statement $(i x)$ with zero and one limit cycles.

Proposition 3 is proved in section 4 .

It remains the open question to know if there are quadratic systems satisfying the assumption of statement (viii) without limit cycles.

Remark 4. In the appendix we present 14 classes of quadratic systems for which it is known that at most have one limit cycle surrounding the origin. We have pass these classes to the Bautin's normal form and we have checked that those quadratic systems do not satisfy the conditions (viii) and (ix) of Theorem 1. Consequently, the results on the uniqueness of limit cycles provided for conditions (viii) and (ix) of Theorem 1 look new.

\section{Preliminary Results}

In this section we recall some basic results that we shall need for proving our Theorem 1.

The next two results correspond to Theorems 2 and 3 of Lloyd [13].

Lemma 5. We have a differential system in polar coordinates

$$
\begin{aligned}
& \dot{r}=F(r, \theta), \\
& \dot{\theta}=G(r, \theta),
\end{aligned}
$$

defined in a simply connected open set $U$ containing the origin, where $F$ and $G$ are $C^{1} 2 \pi$-periodic functions such that $F(0, \theta)=0$ for all $\theta$, and $G(r, \theta)>0$ in $U$. Then, in $U$ the differential system (6) is equivalent to the differential equation

$$
\frac{d r}{d \theta}=\frac{F(r, \theta)}{G(r, \theta)}=S(r, \theta)
$$

Therefore, if

$$
\frac{\partial S}{\partial r} \not \equiv 0
$$

and

$$
\text { either } \frac{\partial S}{\partial r} \leq 0, \quad \text { or } \frac{\partial S}{\partial r} \geq 0 \quad \text { in } U,
$$

the differential system (6) has no limit cycles in $U$.

Remark 6. We note that in [13] the inequalities (9) appear without the equal, but checking the proof of Theorem 2 of [13] we see that it also works under the conditions (8) and (9). 
Lemma 7. Consider the differential system (6) defined in an annular region $\mathcal{A}$ which encircles the origin and where $G(r, \theta)>0$. Then, in $\mathcal{A}$ the differential system (6) is equivalent to the differential equation (7). If (8) and (9) hold in $\mathcal{A}$, then the differential system (6) has at most 1 limit cycle in $\mathcal{A}$.

The remark 6 applies to Lemma 7 but now using the proof of Theorem 3 of [13].

Lemma 8. Under the assumptions of Lemma 7 if $\partial^{3} S / \partial r^{3} \geq 0$ in $\mathcal{A}$, then the differential system (6) has at most 3 limit cycles in $\mathcal{A}$.

Again Remark 6 applies to Lemma 8 but now using the proof of Theorem 8 of [13].

The following two results are well known, for a proof see for instance Gasull [8]. Another proof of the next result can be found in Theorem 1 of Lins Neto [12].

Lemma 9. The Riccati differential equation

$$
\frac{d r}{d \theta}=a(\theta) r^{2}+b(\theta) r+c(\theta)
$$

where $a(\theta), b(\theta)$ and $c(\theta)$ are continuous $2 \pi$-periodic functions, has at most two periodic solutions.

The next result is Theorem 2 of Lins Neto [12] when $a(\theta)>0$, see also Pliss [14]. When $a(\theta) \geq 0$ but $a(\theta) \not \equiv 0$ it is proved in Theorem 8 of $[6]$.

Lemma 10. The Abel differential equation

$$
\frac{d r}{d \theta}=a(\theta) r^{3}+b(\theta) r^{2}+c(\theta) r+d(\theta),
$$

where $a(\theta), b(\theta), c(\theta)$ and $d(\theta)$ are continuous $2 \pi$-periodic functions, and $a(\theta) \geq 0$ for all $\theta$ but $a(\theta) \not \equiv 0$, has at most three periodic solutions.

The next result is due to Bautin [2]

Lemma 11. The Liapunov constants of the quadratic differential system (1) when $\lambda_{1}=0$ are

$$
\begin{aligned}
& V_{3}=-\frac{\pi}{4} \lambda_{5}\left(\lambda_{3}-\lambda_{6}\right), \\
& V_{5}=\frac{\pi}{24} \lambda_{2} \lambda_{4}\left(\lambda_{3}-\lambda_{6}\right)\left(\lambda_{4}+5\left(\lambda_{3}-\lambda_{6}\right)\right), \\
& V_{7}=-\frac{5 \pi}{32} \lambda_{2} \lambda_{4}\left(\lambda_{3}-\lambda_{6}\right)^{2}\left(\lambda_{6}\left(\lambda_{3}-2 \lambda_{6}\right)-\lambda_{2}^{2}\right),
\end{aligned}
$$


where the expression of $V_{5}$ is given when $V_{3}=0$, and the expression of $V_{7}$ is given when $V_{3}=V_{5}=0$. Moreover, the quadratic system (1) has a center at the origin if and only if $\lambda_{1}=V_{3}=V_{5}=V_{7}=0$.

\section{Proof of Theorem 1}

We prove Theorem 1 statement by statement.

Proof of statement $(i)$ of Theorem 1. Since $f=0$ then $d r / d \theta$ does not change sign. If $\lambda_{1} \neq 0$ the solutions $r(\theta)$ of (4) increases or decreases, so these solutions cannot be periodic in the region $C$, and consequently the quadratic system (1) has no limit cycles surrounding the origin.

If $\lambda_{1}=0$ then $d r / d \theta \equiv 0$ and all the solutions in the region $C$ are periodic and circular (except the equilibrium point at the origin), so the system has no isolated periodic orbits surrounding the origin, i.e. no limit cycles surrounding the origin. So statement $(i)$ is proved.

Proof of statement (ii) of Theorem 1. Since $f-\lambda_{1} g=0$ we have that $d r / d \theta=\lambda_{1} r$. Now the proof ends following the same arguments than in the proof of statement $(i)$.

Proof of statement (iii) of Theorem 1. If $g=0$ then the differential equation (4) becomes $d r / d \theta=\lambda_{1} r+f r^{2}$, i.e. it is a Riccati differential equation. By Lemma 9 it has at most two periodic solutions.

Since the differential equation (4) is invariant under the changes of variables $(r, \theta) \rightarrow(-r, \theta+\pi)$, it follows that if $r(\theta)$ is a limit cycle of equation (4), then $-r(\theta+\pi)$ is another limit cycle. Note that the differential equation (4) is defined on the annular region $\mathcal{A}=\{(r, \theta)$ : $1+g r>0\}$ of the cylinder $\left\{(r, \theta) \in \mathbb{R} \times \mathbb{S}^{1}\right\}$. Moreover, since $r=0$ always is a periodic solution of equation (4), we get that the differential equation (4) cannot have a periodic solution in the region of the halfcylinder $r>0$ where it is defined, because then it would have at least three periodic solutions in $\mathcal{A}$, and we have proved that at most it has two periodic solutions. Consequently the quadratic system (1) has no limit cycles surrounding the origin. This completes the proof of statement $($ iii).

Proof of statement (iv) of Theorem 1. Easy computations show that there are two classes of quadratic systems satisfying $f-2 \lambda_{1} g-g^{\prime}=0$. These are

$$
\begin{aligned}
& \dot{x}=-y+\frac{\lambda_{4}}{4} x^{2}-2 \lambda_{2} x y-\frac{\lambda_{4}}{4} y^{2}, \\
& \dot{y}=x+\lambda_{2} x^{2}+\frac{\lambda_{4}}{2} x y-\lambda_{2} y^{2},
\end{aligned}
$$


and

$$
\begin{aligned}
& \dot{x}=\lambda_{1} x-y+2 \lambda_{1} \lambda_{2} x^{2}-2 \lambda_{2} x y, \\
& \dot{y}=x+\lambda_{1} y+\lambda_{2} x^{2}+2 \lambda_{1} \lambda_{2} x y-\lambda_{2} y^{2} .
\end{aligned}
$$

Then using Lemma 11 it follows that all the Liapunov constants of systems (10) and (11) with $\lambda_{1}=0$ are zero. So these systems have a center at the origin, and then it is known that there is no limit cycles surrounding the origin.

Now it remains to prove that system (11) with $\lambda_{1} \neq 0$ has no limit cycles. Indeed, this system has additionally to the focus at the origin a second equilibrium point $(x, y)=\left(-1 / \lambda_{1},-\lambda_{1} / \lambda_{2}\right)$. We translate this equilibrium at the origin doing the change of variables $x=X-1 / \lambda_{1}$ and $y=Y-\lambda_{1} / \lambda_{2}$, obtaining the system

$$
\begin{aligned}
& \dot{X}=-\lambda_{1} X+Y+2 \lambda_{1} \lambda_{2} X^{2}-2 X Y \lambda_{2}, \\
& \dot{Y}=-\left(1+2 \lambda_{1}^{2}\right) X+\lambda_{1} Y+\lambda_{2} X^{2}+2 \lambda_{1} \lambda_{2} X Y-\lambda_{2} Y^{2} .
\end{aligned}
$$

The eigenvalues at the origin of system (12) are $\pm \sqrt{1+\lambda_{1}^{2}} i$. We write the linear part of system (12) into its real Jordan normal form doing the change of variables

$$
\left(\begin{array}{c}
X \\
Y
\end{array}\right)=\left(\begin{array}{cc}
0 & 1 \\
\sqrt{1+\lambda_{1}^{2}} & \lambda_{1}
\end{array}\right)\left(\begin{array}{l}
u \\
v
\end{array}\right)
$$

In the variables $(u, v)$ system $(12)$ becomes

$$
\begin{aligned}
& \dot{u}=-\sqrt{\lambda_{1}^{2}+1} v-\sqrt{\lambda_{1}^{2}+1} u^{2}+2 \lambda_{1} u v+\sqrt{\lambda_{1}^{2}+1} v^{2}, \\
& \dot{v}=\sqrt{\lambda_{1}^{2}+1}(u-2 u v) .
\end{aligned}
$$

Now if we compute the Liapunov constants for this system, which is in the normal form of Bautin, using Lemma 11 we get that the three are zero, so this system at the origin has a center. Since quadratic systems having a center have no limit cycles, this completes the proof of statement $(i v)$.

Proof of statement $(v)$ of Theorem 1. Again easy computations show that there are two classes of quadratic systems satisfying $\lambda_{1} g-9 f=0$. These are

$$
\begin{aligned}
& \dot{x}=-y+y^{2}, \\
& \dot{y}=x-x y,
\end{aligned}
$$




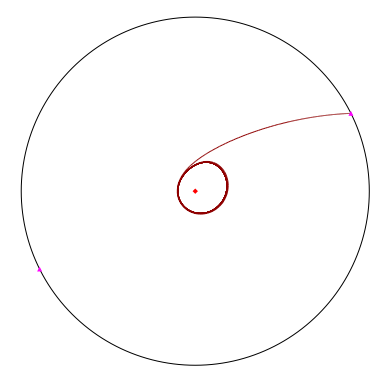

FiguRE 1. Phase portrait of the quadratic system (22) with $\lambda_{1}=1 / 10$.

and

$$
\begin{aligned}
& \dot{x}=\lambda_{1} x-y+\frac{\lambda_{1}}{9} x^{2}-2 y x+\frac{9}{\lambda_{1}} y^{2}, \\
& \dot{y}=x+\lambda_{1} y+x^{2}+\frac{\lambda_{1}^{2}-81}{9 \lambda_{1}} x y-y^{2},
\end{aligned}
$$

with $\lambda_{1} \neq 0$.

By Lemma 11 the quadratic system (13) has a center at the origin, and consequently this system has no limit cycles.

If we write system (14) in polar coordinates and we take $\theta$ as new independent variable we obtain the differential equation

$$
\frac{d r}{d \theta}=\frac{\lambda_{1} r\left(9 \lambda_{1}+\lambda_{1} r \cos \theta-9 r \sin \theta\right)}{9\left(\lambda_{1}+\lambda_{1} r \cos \theta-9 r \sin \theta\right)}=S(r, \theta),
$$

defined in the simply connected region $C$. Since

$$
\frac{\partial S}{\partial r}=\frac{\lambda_{1}}{9}\left(\frac{8 \lambda_{1}^{2}}{\left(\lambda_{1}+\lambda_{1} r \cos \theta-9 r \sin \theta\right)^{2}}+1\right)
$$

is $\geq 0$ if $\lambda_{1}>0$, or $\leq 0$ if $\lambda_{1}<0$, we have that $\partial S / \partial r$ satisfies (8) and (9) in the region $C$, and consequently, we can apply Lemma 5 , to the differential equation (15), and the proof of statement $(v)$ follows.

Proof of statement (vi) of Theorem 1. After easy computations the quadratic systems satisfying $9 \lambda_{1} g-f=0$ are system (13) and the systems

$$
\begin{aligned}
& \dot{x}=\lambda_{1} x-y+9 \lambda_{1} x^{2}-2 x y+\frac{1}{9 \lambda_{1}} y^{2}, \\
& \dot{y}=x+\lambda_{1} y+x^{2}+\frac{81 \lambda_{1}^{2}-1}{9 \lambda_{1}} x y-y^{2},
\end{aligned}
$$


with $\lambda_{1} \neq 0$. Since we have proved that system (13) has no limit cycles, it only remains to prove that system (16) has no limit cycles.

Writing system (16) in polar coordinates and taking $\theta$ as the new independent variable we have the differential equation

$$
\frac{d r}{d \theta}=\frac{\lambda_{1} r+r^{2}\left(9 \lambda_{1} \cos \theta-\sin \theta\right)}{1+r\left(\cos \theta-\sin \theta /\left(9 \lambda_{1}\right)\right)}=S(\theta, r) .
$$

Then

$$
\frac{\partial^{3} S}{\partial r^{3}}=-\frac{3888 \lambda_{1}^{3}\left(9 \lambda_{1} \cos \theta-\sin \theta\right)^{2}}{\left(9 \lambda_{1}+r\left(9 \lambda_{1} \cos \theta-\sin \theta\right)\right)^{4}} .
$$

We can assume that $\lambda_{1}<0$, otherwise we reverse the sign of the time in the differential system (16). Therefore we have that $\partial^{3} S / \partial r^{3} \geq 0$, and the equality holds at only finitely many points. Taking into account $C$, which is defined in (3), we know that equation (17) is defined on an annular region, saying $\mathcal{A}$, of the cylinder $\left\{(\theta, r) \in \mathbb{S}^{1} \times \mathbb{R}\right\}$, see Fig. 2 .

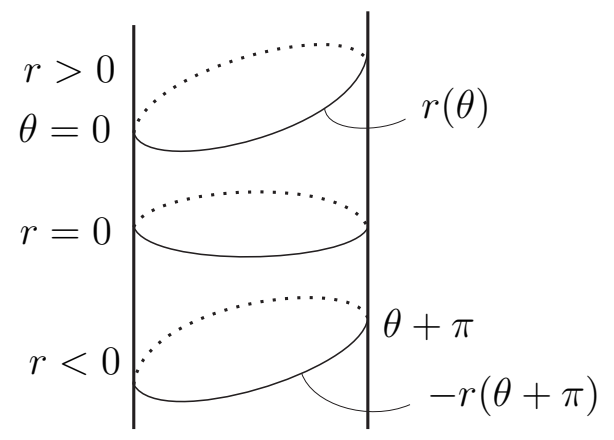

FiguRE 2. Illustration of the three periodic orbits on the cylinder.

Taking $\theta=0$ as a Poincaré section of equation (17) in $\mathcal{A}$, and $r_{0} \in \mathbb{R}$ as an initial point of equation (17) at $\theta=0$. Let $H\left(r_{0}\right)=r\left(2 \pi, r_{0}\right)-$ $r_{0}$ be the displacement function, with $r\left(\theta, r_{0}\right)$ being the solution of equation (17) such that $r\left(0, r_{0}\right)=r_{0}$ for the point $\left(0, r_{0}\right) \in \mathcal{A}$. Since $\partial^{3} S / \partial r^{3} \geq 0$ and it is not identically zero, from the proof of Theorem 8 of [13] we have that $H^{\prime \prime \prime}\left(r_{0}\right)>0$ for all $r_{0}$ where the map $H$ is defined. The number of zeros of $H\left(r_{0}\right)$ taking into account their multiplicities cannot be larger than 3 , otherwise $H^{\prime \prime \prime}\left(r_{0}\right)$ would have some zero, a contradiction. So equation (17) has at most three different zeros with multiplicity one.

We saw in the proof of statement (iii) that if $r(\theta)$ is a solution of the differential equation (4), then $-r(\theta+\pi)$ is another solution. Moreover, since $r=0$ always is a periodic solution of equation (17) 
(see Fig. 2), we get that when equation (17) has three simple zeros, it has a unique positive zero. Consequently, the quadratic polynomial differential system (16) has at most one limit cycle in $r>0$. Moreover, if the limit cycle exists it is hyperbolic, because then the zeros of $H(x)$ are simple, for a definition of a hyperbolic limit cycle see for instance the Chapter 8 of [7].

Now we shall prove that the quadratic systems (16) have no limit cycles. Indeed, systems (16) with $\lambda_{1} \neq 0$ have a unique finite equilibrium, a hyperbolic focus; and have a unique pair of infinite equilibria a semi-hyperbolic saddle-node, having only one separatrix $\gamma$ outside the infinity, which by the Poincaré-Bendixson Theorem must go to surround the unstable focus, see for more details on the PoincaréBendixson Theorem section 1.7 of [7]. Moreover using Theorem 2.19 of [7] it follows that if the origin is a stable (respectively unstable) focus, then the separatrix $\gamma$ is unstable (respectively stable). Therefore, since system (16) has at most one limit cycle, if such a limit cycle exists it must be semistable, in contradiction with the fact that if the limit cycle exists, it must be hyperbolic, which we proved in the previous paragraph. This completes the proof of statement $(v i)$.

Proof of statement (vii) of Theorem 1. Doing the change of variables $R=\sqrt{r}$ in the region $C$, the differential equation (4) becomes

$$
\frac{d R}{d \theta}=\frac{\lambda_{1} R+f R^{3}}{2\left(1+g R^{2}\right)}=S(R, \theta) .
$$

Clearly the image of the simply connected region $C$ under the map $r \rightarrow \sqrt{r}=R$ is another simply connected region $S$ containing the origin $R=0$.

We have

$$
\frac{\partial S}{\partial R}=\frac{\lambda_{1}+\left(3 f-\lambda_{1} g\right) R^{2}+f g R^{4}}{2\left(1+g R^{2}\right)^{2}} .
$$

Then, clearly if $\left(3 f-\lambda_{1} g\right)^{2}-4 \lambda_{1} f g=\left(\lambda_{1} g-f\right)\left(\lambda_{1} g-9 f\right) \leq 0$, we have that $\partial S / \partial R$ satisfies (8) and (9) in the region $S$, and consequently, we can apply Lemma 5 , to the differential equation (18), and the proof of statement (vii) follows.

Proof of statement (viii) of Theorem 1. Doing the change of variables $R=1 / \sqrt{r}$ in the region $C$, the differential equation (4) becomes

$$
\frac{d R}{d \theta}=-\frac{\lambda_{1} R^{3}+f R}{2\left(g+R^{2}\right)}=S(R, \theta) .
$$

Now the image of the region $C$ by the map $r \rightarrow 1 / \sqrt{r}=R$ is an annular region $\mathcal{A}$, and one of the boundaries of this annulus is the infinity. 
We get

$$
\frac{\partial S}{\partial R}=-\frac{f g-\left(f-3 \lambda_{1} g\right) R^{2}+\lambda_{1} R^{4}}{2\left(g+R^{2}\right)^{2}} .
$$

Then, clearly if $\left(f-3 \lambda_{1} g\right)^{2}-4 \lambda_{1} f g=\left(\lambda_{1} g-f\right)\left(9 \lambda_{1} g-f\right) \leq 0$, we have that $\partial S / \partial R$ satisfies conditions (8) and (9) in the annular region $\mathcal{A}$, and consequently we can apply Lemma 7 , to the differential equation (19), and this completes the proof of statement (viii).

Proof of statement (ix) of Theorem 1. Note that $g\left(\lambda_{1} g-f\right)$ is the coefficient of $\rho^{3}$ in the Abel differential equation (5).

Assume that $g\left(\lambda_{1} g-f\right) \geq 0$ and $g\left(\lambda_{1} g-f\right) \not \equiv 0$. Therefore, by Lemma 10 the Abel differential equation (5) has at most three periodic solutions, and consequently the differential equation (4) has at most three periodic solutions.

We saw in the proof of statement (iii) that if $r(\theta)$ is a solution of the differential equation (4), then $-r(\theta+\pi)$ is another solution. Moreover, since $r=0$ always is a periodic solution of equation (4), we get that the differential equation (4) has at most one periodic solution in the region of the half-cylinder $r>0$ where it is defined. Consequently the quadratic system (1) has at most one limit cycle. This completes the proof of statement $(i x)$.

\section{Proof of Proposition 3}

We prove Proposition 3 following the statements of Theorem 1.

Examples of statement $(i)$. It is easy to compute that all quadratic systems (1) with $f=0$ are

$$
\begin{aligned}
& \dot{x}=\lambda_{1} x-y-\lambda_{4} y^{2}, \\
& \dot{y}=x+\lambda_{1} y+\lambda_{4} x y .
\end{aligned}
$$

Examples of statement (ii). Again it is easy to verify that all quadratic systems (1) with $f-\lambda_{1} g=0$ are the systems (20) and also the systems

$$
\begin{aligned}
& \dot{x}=\lambda_{1} x-y+\lambda_{1} \lambda_{2} x^{2}-2 \lambda_{2} x y+\frac{\lambda_{2}}{\lambda_{1}} y^{2}, \\
& \dot{y}=x+y \lambda_{1}+\lambda_{2} x^{2}+\frac{\left(\lambda_{1}-1\right)\left(\lambda_{1}+1\right) \lambda_{2}}{\lambda_{1}} x y-\lambda_{2} y^{2} .
\end{aligned}
$$

Examples of statement (iii). Quadratic systems (1) having $g=0$ are

$$
\begin{aligned}
& \dot{x}=\lambda_{1} x-y-\lambda_{3} x^{2}, \\
& \dot{y}=x+\lambda_{1} y-\lambda_{3} x y .
\end{aligned}
$$


Examples of statements $(i v),(v)$ and $(v i)$. These examples are given in the proofs of the corresponding statements $(i v),(v)$ and $(v i)$ of Theorem 1.

Examples of statement (vii). For the quadratic systems

$$
\begin{aligned}
& \dot{x}=\lambda_{1} x-y+\frac{1}{4 \lambda_{1}^{2}}\left(\lambda_{1} x-5 y\right)^{2}, \\
& \dot{y}=x+\lambda_{1} y+\frac{1}{4 \lambda_{1}^{2}}\left(\lambda_{1} x-5 y\right)\left(5 x+\lambda_{1} y\right),
\end{aligned}
$$

depending on the parameters $\lambda_{1}$. We have that

$$
\left(\lambda_{1} g-f\right)\left(\lambda_{1} g-9 f\right)=-\frac{\left(\lambda_{1} \cos \theta-5 \sin \theta\right)^{2}}{\lambda_{1}^{2}} \leq 0 .
$$

Therefore there are quadratic systems satisfying the assumptions of statement (vii).

Now the proof that quadratic systems (21) have no limit cycles is identical to the proof showing that systems (16) have no limit cycles.

Examples of statement (viii). We consider the family of quadratic systems

$$
\begin{aligned}
& \dot{x}=\lambda_{1} x-y+\frac{1}{5 \lambda_{1}}\left(5 \lambda_{1} x-y\right)^{2}, \\
& \dot{y}=x+\lambda_{1} y+\frac{1}{5 \lambda_{1}}\left(5 \lambda_{1} x-y\right)\left(x+5 \lambda_{1} y\right),
\end{aligned}
$$

depending on the parameters $\lambda_{1}$. We have that

$$
\left(\lambda_{1} g-f\right)\left(9 \lambda_{1} g-f\right)=-\frac{16}{25}\left(\sin \theta-5 \lambda_{1} \cos \theta\right)^{2} \leq 0 .
$$

Hence this family of quadratic systems satisfy the hypotheses of statement (viii).

The quadratic system (22) with $\lambda_{1} \neq 0$ has a unique finite equilibrium, a hyperbolic focus; and has a unique pair of infinite equilibria a semi-hyperbolic saddle-node, having only one separatrix $\gamma$ outside the infinity, which by the Poincaré-Bendixson Theorem must go to surround the unstable focus. Moreover using Theorem 2.19 of [7] it follows if the origin is a stable (respectively unstable) focus, then the separatrix $\gamma$ is stable (respectively unstable). So again by the Poincaré-Bendixson Theorem at least one limit cycle surrounds such unstable focus localized at the origin of coordinates, see Figure 2. Then, by statement (viii) of Theorem 1 this limit cycle is unique. 
In short, we have proved that there are quadratic systems satisfying the assumptions of statement (viii) of Theorem 1 having one limit cycle surrounding the origin.

Examples of statement $(i x)$. For the quadratic systems

$$
\begin{aligned}
& \dot{x}=\lambda_{1} x-y-b x^{2}-\varepsilon x y, \\
& \dot{y}=x+\lambda_{1} y-b x y,
\end{aligned}
$$

depending of three parameters $\lambda_{1}, \varepsilon$ and $b$ we have that

$$
g\left(\lambda_{1} g-f\right)=\varepsilon b \cos ^{2} \theta \sin ^{2} \theta+\varepsilon^{2} \cos ^{2} \theta \sin ^{3} \theta\left(\cos \theta+\lambda_{1} \sin \theta\right) .
$$

So if the parameter $\varepsilon$ is sufficiently small and $\varepsilon b>0$ we have that $g\left(\lambda_{1} g-f\right) \geq 0$, and of course $g\left(\lambda_{1} g-f\right) \not \equiv 0$. So the quadratic system (23) satisfies the assumption of statement (viii) of Theorem 1, hence it has at most one limit cycle.

The eigenvalues at the origin of system (23) are $\lambda_{1} \pm i$. Therefore when $\lambda_{1}=0$ if there is a Liapunov constant which is not zero, then a Hopf bifurcation of an infinitesimal periodic orbit takes place at the origin of coordinates, see for more details about Hopf bifurcations [10]. The Liapunov constants for the quadratic differential systems (1) with $\lambda_{1}=0$ were computed by Bautin [2] and the first one is

$$
V_{3}=-\frac{\pi}{4} \lambda_{5}\left(\lambda_{3}-\lambda_{6}\right),
$$

see Lemma 11. Then, for our system (23) when $\lambda_{1}=0$, we have $V_{3}=\varepsilon b \pi / 4>0$ because $\varepsilon b>0$. Hence there are systems (23) with $\varepsilon b>0$ and $\varepsilon$ sufficiently small such that in a neighborhood of $\lambda_{1}=0$ exhibit zero limit cycles or one limit cycle.

In short we have proved that there are quadratic systems satisfying the conditions of statement $(i x)$ of Theorem 1 exhibiting either zero, or one limit cycle surrounding the origin.

\section{Appendix}

In the following we provide a list with 14 classes of quadratic differential systems for which are known that at most have one limit cycle surrounding the origin. The first 12 come from the section 9 of the Ye's book [21], the last two classes come from the articles of Zhang [22], and of Sun [19], respectively.

(i) The quadratic systems in the Ye's normal form $(I)$ :

$$
\dot{x}=-y+\delta x+l x^{2}+x y+n y^{2}, \quad \dot{y}=x .
$$


(ii) The quadratic systems in the Ye's normal form $(I I I)_{n=0}$ :

$$
\dot{x}=-y+l x^{2}+m x y, \quad \dot{y}=x+a x^{2}+b x y,
$$

having a weak focus of order 2 at the origin, i.e. there satisfy the conditions:

$$
\begin{aligned}
m l-a(b+2 l) & =0, \\
m a(5 a-m)\left(b l^{2}-a^{2}(b+2 l)\right) & \neq 0 .
\end{aligned}
$$

(iii) The quadratic systems in the Ye's normal form $(I I I)_{n \neq 0}$ :

$$
\dot{x}=-y+l x^{2}+m x y+y^{2}, \quad \dot{y}=x+a x^{2}+b x y,
$$

having a weak focus of order 2 at the origin, i.e. there satisfy the conditions:

$$
\begin{aligned}
m(l+1)-a(b+2 l) & =0, \\
m a(5 a-m)\left((b+1)(l+1)^{2}-a^{2}(b+2 l+1)\right) & \neq 0 .
\end{aligned}
$$

(iv) The quadratic systems in the Ye's normal form $(I I I)_{a=0}$ :

$$
\dot{x}=-y+\delta x+l x^{2}+m x y+n y^{2}, \quad \dot{y}=x+b x y .
$$

(v) A special quadratic systems in the Ye's normal form (III):

$$
\dot{x}=-y+\delta x+n y^{2}, \quad \dot{y}=x+a x^{2}-x y,
$$

with $0<n<1$.

(vi) Quadratic systems having a degenerate finite singularity can be written in the Ye's normal form $(I I I)$ :

$$
\begin{aligned}
& \dot{x}=-y+\delta x+l x^{2}-\delta x y+y^{2}, \\
& \dot{y}=x+a x^{2}-x y, \quad|\delta|<2,
\end{aligned}
$$

which has $(0,1)$ as a degenerate singularity (i.e. the linearization of the system at $(0,1)$ has two zero eigenvalues and it is not zero), and satisfying $a \delta(2 l-1) \neq 0$ (otherwise, the origin is a center).

(vii) Quadratic systems having a degenerate singularity at infinity can be written in the Ye's normal form $(I I I)$ :

$$
\dot{x}=-y+\delta x+l x^{2}, \quad \dot{y}=x+a x^{2}+b x y, \quad|\delta|<2 .
$$

(viii) A quadratic system in the Ye's normal form (III):

$$
\dot{x}=-y+\delta x(y-1)+l x^{2}+n y^{2}, \quad \dot{y}=x+a x^{2}-x y,
$$

with $1 / 2 \leq n<1$ (otherwise it is unknown the existence or not of limit cycles). 
(ix) A quadratic system in the Ye's normal form $(I I I)$ :

$$
\dot{x}=-y+\delta x(y-1)+l x^{2}+y^{2}, \quad \dot{y}=x+a x^{2}+b x y,
$$

with $\delta \neq 0, a>0, b+1<0$.

$(x)$ Quadratic systems having a finite singularity of multiplicity $\geq 3$ can be written in the Ye's normal form (III):

$\dot{x}=-y+\delta x+l x^{2}-(a+\delta) x y+y^{2}, \quad \dot{y}=x+a x^{2}-x y$,

which have $(0,1)$ as a singularity of multiple 3 , and satisfy $\delta>$ and $0<a \delta / l<1$ (otherwise the systems have no limit cycles).

(xi) Quadratic systems having a singularity of multiplicity $\geq 3$ at infinity can be written in the Ye's normal form (III):

$$
\dot{x}=-y+\delta x+l x^{2}+m x y+n y^{2}, \quad \dot{y}=x+b x^{2}+c x y,
$$

with coefficients satisfying

- either $b=l \neq 0, m=c$;

- or $l=0, m=b \delta, b \neq 0$.

(xii) The quadratic systems in the Ye's normal form $(I I I)$ :

$$
\begin{aligned}
& \dot{x}=-y+\delta x+(m-b \delta) x^{2}+b x y, \\
& \dot{y}=x+\left(n+2 \delta m-b \delta^{2}\right) x^{2}+(b \delta-m) x y,
\end{aligned}
$$

with coefficients satisfying either $b=0$; or $m^{2}+b n=0$.

The quadratic systems

$$
\dot{x}=-y+\delta x+l x^{2}+n y^{2}, \quad \dot{y}=x+a x^{2}-x y,
$$

have at most one limit cycle under the conditions

(xiii) either $a<0, l>1 / 2,0<n<1$ and $\delta<0$;

(xiv) or $0<n \leq 1 / 2(1 / 2 \leq n<1),-n a^{2}<l<1 / 2, a \delta<0(>0)$.

There are some other results on the uniqueness of limit cycles of quadratic systems that we do not list here.

\section{ACKNOWLEDGMENTS}

The first author is partially supported by a MINECO/FEDER grant MTM2008-03437, a CIRIT grant number 2009SGR-410, an ICREA Academia, two grants FP7-PEOPLE-2012-IRSES 316338 and 318999, the grant UNAB13-4E-1604, and from the recruitment program of high-end foreign experts of China. 
The second author is partially supported by NNSF of China grant number 11271252, by RFDP of Higher Education of China grant number 20110073110054, and by FP7-PEOPLE-2012-IRSES-316338 of Europe, and by innovation program of Shanghai municipal education commission grant 15ZZ012.

This work was done during the visit of the first author to Shanghai Jiao Tong University, who appreciate its support and hospitality.

\section{REFERENCES}

[1] A.A. Andronov, Les cycles limites de Poincaré et la théorie des oscillations auto-entretenues, C.R. Acad. Sci. Paris 89 (1929), 559-561.

[2] N.N. BAUTIN, On the number of limit cycles which appear with the variation of the coefficients from an equilibrium position of focus or center type, Math. USSR-Sb. 100 (1954), 397-413.

[3] M. CARBonell AND J. Llibre, Limit cycles of a class of polynomial systems, Proc. Royal Soc. Edinburgh 109A (1988), 187-199.

[4] M. Carbonell and J. Llibre, in Hopf bifurcation, averaging methods and Liapunov quantities for polynomial systems with homogeneous nonlinearities, Proc. European Conference on Iteration Theory, ECIT87, World Scientific, Singapore, 1989, pp. 145-160.

[5] L.A. Cherkas, Number of limit cycles of an autonomous second-order system, Differential Equations 5 (1976), 666-668.

[6] B. Coll, A. Gasull And J. Llibre, Some theorems on the existence, uniqueness and non existence of limit cycles for quadratic systems, J. Differential Equations 67 (1987), 372-399.

[7] F. Dumortier, J. Llibre And J.C. Artés, Qualitative theory of planar differential systems, Universitext, Springer-Verlag, 2006.

[8] A. Gasull, From Abel's differential equations to Hilbert's sixteenth problem, (Catalan) Butl. Soc. Catalana Mat. 28 (2013), no. 2, 123-146.

[9] A. Gasull And J. Llibre, Limit cycles for a class of Abel equations, SIAM J. Math. Anal. 21 (1990), 1235-1244.

[10] Y. Kuznetsov, Elements of applied bifurcation theory, Applied Mathematical Sciences, Vol. 112, Springer-Verlag, New York, 2004.

[11] A. LiÉnARD, Etude des oscillations entretenues, Rev. Générale de l'Electricité 23 (1928), 901-912.

[12] A. Lins Neto, On the number of solutions of the equation $d x / d t=$ $\sum_{j=0}^{n} a_{j}(t) x^{j}, 0 \leq t \leq 1$, for which $x(0)=x(1)$, Invent. Math. 59 (1980), $67-76$.

[13] N.G. LlOYD, A note on the number of limit cycles in certain two-dimensional systems, J. London Math. Soc. 20 (1979), 277-286.

[14] V.A. PLiss, Non-local problems of the Theory of Oscillations, Academic Press, New York, 1966.

[15] H. Poincaré, Mémoire sur les courbes définies par une équation differentielle I, II, J. Math. Pures Appl. 7 (1881), 375-422; 8 (1882), 251-296; Sur les courbes définies pas les équations differentielles III, IV, 1 (1885), 167-244; 2 (1886), $155-217$.

[16] VAN DER PoL, On relaxation-oscillations, Phil. Mag. 2 (1926), 978-992. 
[17] J.W. REYN, Phase portraits of planar quadratic systems, Mathematics and Its Applications (Springer), 583, Springer, New York, 2007.

[18] D. Schlomiuk, Algebraic particular integrals, integrability and the problem of the center, Trans. Amer. Math. Soc. 338 (1993), 799-841.

[19] JiAnHuA Sun, The uniqueness and bifurcation of limit cycles of quadratic systems $(I I I)_{m=0}(0<n<1)$, Chinese Sci. Bull. 37 (1992), 330.

[20] Ye Yanqian et al., Theory of Limit Cycles, Transl. Math. Monographs 66, Amer. Math. Soc., Providence, 1984.

[21] Ye YanqIan, Qualitative Theory of Polynomial Differential Systems, Shanghai Science \& Technology Pub., Shanghai, 1995.

[22] Xiang Zhang, The uniqueness of limit cycles of quadratic systems $(I I I)_{m=0}$, Chinese Sci. Bull. 42 (1997), 351-352.

${ }^{1}$ Departament de Matemàtiques, Universitat Autònoma de Barcelona, 08193 Bellaterra, Barcelona, Catalonia, Spain

E-mail address: jllibre@mat.uab.cat

2 Department of Mathematics, MOE-LSC, Shanghai Jiao tong University, Shanghai, 200240, P. R. China

E-mail address: xzhang@sjtu.edu.cn 